\title{
Tamsulosin for treatment of unilateral distal ureterolithiasis: a systematic review and meta- analysis
}

\author{
Christian Malo, MD*; Jean-Sébastien Audette-Côté, MD*; Marcel Émond, $\mathrm{MD}^{*}{ }^{\dagger}$; \\ Alexis F. Turgeon, $\mathrm{MD}^{\dagger \neq}$
}

\section{ABSTRACT}

Objectives: The lifetime prevalence of ureterolithiasis is approximately $13 \%$ for men and $7 \%$ for women in the United States. Tamsulosin, an $\alpha$-antagonist, has been used as therapy to facilitate the expulsion of lithiasis. Whether it is a good treatment for distal lithiasis remains controversial. We conducted a systematic review and meta-analysis to evaluate the effect of tamsulosin on the passage of distal ureterolithiasis.

Methods: A systematic search was conducted using MEDLINE, EMBASE, and Cochrane Central. Trial eligibility was evaluated by two investigators. All randomized controlled trials (RCTs) comparing tamsulosin to standard therapy or placebo for the treatment of a single distal ureterolithiasis $\leq 10 \mathrm{~mm}$ in adult patients with renal colic confirmed by radiographic imaging were included. Data extraction was conducted in duplicate. Primary outcome was the expulsion rate, and secondary outcomes were the mean time for ureterolithiasis expulsion, analgesic requirements, and side effects. Mantel-Haenszel random effect models were used, and heterogeneity was assessed using $1^{2}$ statistics. Data were presented with relative risks (RRs).

Results: The search strategy identified 685 articles, of which 22 studies were included. Combined results suggested a benefit for the expulsion of ureterolithiasis $(\leq 10 \mathrm{~mm})$ when tamsulosin was used compared to a standard treatment (RR 1.50 [95\% Cl 1.31-1.71], $\mathrm{I}^{2}=70 \%$ ). A decrease in the average time of expulsion of the ureterolithiasis of 3.33 days in favour of tamsulosin was observed $\left.(95 \% \mathrm{Cl}-4.23,-2.44], \mathrm{I}^{2}=67 \%\right)$. Conclusion: Tamsulosin increases the rate of spontaneous passage of distal ureterolithiasis $(\leq 10 \mathrm{~mm}$ ).

\section{RÉSUMÉ}

Objectif: La prévalence de la lithiase urétérale au cours de la vie est d'environ $13 \%$ chez les hommes et $7 \%$ chez les femmes aux États-Unis. La tamsulosine, un alpha-bloquant, est utilisée comme traitement pour faciliter I'expulsion des calculs; toutefois, le recours à ce traitement dans la lithiase distale fait l'objet de controverse. Nous avons donc mené un examen méthodique et une méta-analyse afin d'évaluer l'effet de la tamsulosine sur le passage des lithiases urétérales distales.

Méthode: Une recherche méthodique a été effectuée dans les bases de données MEDLINE, EMBASE, et Cochrane Central, et l'acceptabilité des essais a été soumise à l'examen de deux chercheurs. Ont été retenus tous les essais comparatifs, à répartition aléatoire, visant à mettre en parallèle la tamsulosine avec les procédés thérapeutiques habituels ou avec un placébo dans le traitement d'une seule lithiase urétérale distale, $\leq 10 \mathrm{~mm}$, confirmée à la radiographie, chez des adultes souffrant de colique néphrétique. L'extraction des données a été réalisée en double. Le principal critère d'évaluation était le taux d'expulsion, et les critères d'évaluation secondaires portaient sur le temps moyen écoulé avant l'expulsion des lithiases urétérales, I'administration d'analgésiques, et les effets indésirables. Nous avons eu recours aux modèles à effets aléatoires selon la méthode de Mantel-Haenszel, et I'hétérogénéité a été évaluée à l'aide du test statistique $\mathrm{I}^{2}$. Les données sont exprimées sous forme de risque relatif $(R R)$.

Résultats: La stratégie de recherche documentaire a permis de relever 685 articles, et sur ce nombre 22 études ont été retenues. Les résultats globaux semblent indiquer que la tamsulosine a un effet favorable sur l'expulsion des lithiases urétérales ( $\leq 10 \mathrm{~mm}$ ) comparativement au traitement courant (RR 1.50 [IC à 95\% 1.31-1.71]; $\left.\right|^{2}=70 \%$ ). Une diminution de 3.33 jours du temps moyen écoulé avant l'expulsion des lithiases urétérales a également été observée en faveur de la tamsulosine (IC à 95\% $-4.23,-2.44]$; $\left.\right|^{2}=$ $67 \%)$.

From the *Department of Family and Emergency Medicine, Université Laval, Québec City, QC; †Axe de recherche en Traumatologie - Urgence Soins intensifs, Centre de recherche du CHU de Québec (Hôpital de l'Enfant-Jésus), Québec City, QC; †Division of Critical Care Medicine, Department of Anesthesiology, Université Laval, Québec City, OC.

Correspondence to: Dr. Christian Malo, Axe de recherche en Traumatologie-Urgence-Soins intensifs, Centre de recherche du CHU de Québec, Hôpital Enfant-Jésus, 1401, 18ième rue, Québec, QC G1J 1Z4; christian.malo.1@ulaval.ca.

This article has been peer reviewed. 
Conclusion: La tamsulosine augmente le taux d'évacuation spontanée des lithiases urétérales distales ( $\leq 10 \mathrm{~mm}$ ).
Keywords: $\alpha$-antagonist, $\alpha$-blocker, nephrolithiasis, renal colic, tamsulosin, ureterolithiasis
The lifetime prevalence of ureterolithiasis is approximately $13 \%$ for men and $7 \%$ for women in the United States. ${ }^{1}$ From 1994 to 2000, the number of consultations for renal colic almost doubled, and a trend toward an increased prevalence is suspected..$^{2}$ Because of its frequency, this condition leads to significant costs in terms of medical consultations, radiologic examinations, and hospitalizations. ${ }^{3}$ The choice of treatment for renal colic depends on many factors, including the size of the ureterolithiasis. Ureterolithiasis $\leq 4 \mathrm{~mm}$ is spontaneously expelled in $78 \%$ of cases, that of 5 to $7 \mathrm{~mm}$ is expelled in $60 \%$ of cases, and that $\geq 8 \mathrm{~mm}$ is expelled in $39 \%$ of cases. ${ }^{4}$ It is common practice to favour a conservative treatment (observation) for ureterolithiasis of $<7 \mathrm{~mm}$. For ureterolithiasis of larger size, a more invasive urologic treatment is recommended, such as extracorporeal shock wave lithotripsy (ESWL) or ureteroscopy. ${ }^{5}$ These urologic interventions are costly and are associated with a surgical risk. ${ }^{6}$ An overall postureteroscopy complication rate of 10 to $20 \%$ and a rate of major complications of 3 to $5 \%$ have been reported. ${ }^{7}$ Over the past 20 years, different medical therapies have been developed to facilitate the expulsion of ureterolithiasis.

Among recent therapies, tamsulosin is promising because of its ease of administration and cost. Recent meta-analyses observed the efficacy of $\alpha$-antagonist to increase the spontaneous passage of ureterolithiasis $\leq 10 \mathrm{~mm}$ located in the distal ureter..$^{8-11}$ In a study published in 2009, emergency physicians reported prescribing tamsulosin for less than $25 \%$ of their patients with ureterolithiasis, and a comparable proportion said they had never heard of this therapy. ${ }^{12} \mathrm{~A}$ more recent survey observed that only $55 \%$ of emergency physicians reported usually or always using medical expulsive therapy for patients with distal ureterolithiasis $<10 \mathrm{~mm}$. When a medical expulsive therapy was used, tamsulosin was prescribed only about $50 \%$ of the time. ${ }^{13}$ Despite that, the use of tamsulosin still remains controversial because a paucity of available evidence and the poor methodological quality of studies included in previous meta-analyses precluded the consideration of the treatment as a standard of care. A recent large multicentre randomized controlled trial (RCT) showed no significant effect of this therapy, ${ }^{14}$ and numerous new smaller RCTs have been published since the last metaanalysis. ${ }^{8}$

Considering the equipoise surrounding the use of tamsulosin and the amount of research performed on the topic over the last few years, we conducted a systematic review and a meta-analysis of RCTs comparing tamsulosin to standard therapy or placebo for the treatment of distal ureterolithiasis to determine its effect on the spontaneous stone expulsion rate, the mean time to stone expulsion, and adverse events.

\section{METHOD}

\section{Search strategy}

A systematic search was conducted using MEDLINE (1949-March 2012), EMBASE (1974-March 2012), and the Cochrane Central Register of Controlled Trials (1974-March 2012). The references of included studies were searched to identify additional potential relevant studies. No language restriction was applied. Keywords related to urolithiasis, related $\mathrm{MeSH}$ (MEDLINE), and Emtree (EMBASE) were combined in our search strategy (Appendix 1, Appendix 2, and Appendix 3).

\section{Study eligibility}

Two investigators (C.M., J.-S.A.-C.) independently evaluated the eligibility of studies. In case of disagreement, a third party was involved (A.F.T.). To be eligible, the studies had to 1) have randomized patients; 2) compare orally administered tamsulosin (regardless of the dose) to standard therapy (opioids \pm nonsteroidal antiinflammatory drugs [NSAIDs] \pm hydration \pm antibiotics) or to a placebo for the treatment of a single distal ureterolithiasis; and 3) include patients age 18 years or older with a diagnosis of renal colic confirmed by radiologic imaging. The following conditions met our exclusion criteria: 1) a diagnosis of high or low urinary tract infection associated with ureterolithiasis, 2) pregnancy, 3) diabetes, and 4) high blood pressure treated with an $\alpha$-blocker. Studies that were the object of multiple 
duplications were reviewed, and only the publication that brought the most information was included. When data on the size of the ureterolithiasis were not available, the authors were contacted by email. Studies using tamsulosin as a therapy combined with a calcium blocker or a corticosteroid were excluded because the objective of this study was to focus on the effect of tamsulosin alone. ${ }^{8,15-20}$ If a study included several comparison groups, only the results for the groups using tamsulosin and those of the control group were included.

\section{Data abstraction}

A standardized data abstraction form was developed and data from each study were abstracted independently by two investigators (C.M., J.-S.A.-C.). For each of the included studies, the following data were collected: 1) age; 2) sex; 3) size of ureterolithiasis and its location; 4) rates of spontaneous expulsion of ureterolithiasis dichotomized by mean stone size ( $\leq 5 \mathrm{~mm}$ or $>5 \mathrm{~mm}$ ); 5) time to expulsion; 6) side effects associated with the treatment; 7) number of pain episodes; 8) number of visits to the emergency department (ED) for recurrent renal colic; and 9) treatment compliance. The confirmation of expulsion was extracted and categorized as either self-report by a participant or radiologic confirmation (plain radiograph or computed tomographic [CT] scan). Data abstraction for non-English publications was confirmed with the previously published meta-analysis. ${ }^{8}$

\section{Outcomes}

The primary outcome was the percentage of spontaneous passage of distal ureterolithiasis of $\leq 10 \mathrm{~mm}$ comparing tamsulosin to standard treatment. Secondary outcomes were to evaluate the 1) impact of the mean stone size on the spontaneous expulsion rate, 2) mean time for stone expulsion, and 3) complications and side effects associated with this treatment.

\section{Risk of bias assessment}

The risk of bias was evaluated by the one author (C.M.) using the Cochrane risk of bias assessment tool (Appendix 4). ${ }^{21}$ We summarized the methodological quality as 1) low risk of bias if at least four domains of the Cochrane tool were considered at low risk of bias; 2) moderate risk of bias if two to three domains were considered at low risk of bias; or 3) high risk of bias if a study had one or no domain at low risk of bias.

\section{Statistical analysis}

A meta-analysis was conducted using Mantel-Haenszel random effect models. Data were presented using relative risks (RRs) and 95\% confidence intervals (CIs) for dichotomous variables (spontaneous passage of ureterolithiasis $\leq 10 \mathrm{~mm}$ ), whereas mean differences and $95 \%$ CI were used for continuous variables (time to stone expulsion and number of pain episodes). The presence of statistical heterogeneity was assessed with the $\mathrm{I}^{2}$ index and the Cochrane $\mathrm{Q}$ test. Predetermined sensitivity analyses were performed to identify potential sources of heterogeneity based on double-blind randomization (present or absent), methodological quality (low versus high and moderate risk of bias), mean stone size ( $\leq 5 \mathrm{~mm}$ or $>5 \mathrm{~mm})$, dosage $(0.2 \mathrm{mg}$ v. $0.4 \mathrm{mg}$ orally daily), and duration ( $\geq 21$ days or $<21$ days) of treatment. Because we knew that a significant number of new articles have been published on tamsulosin in the past few years, we decided to report separate subgroup analysis of RCTs published since the last meta-analysis and stratified by country of origian. The presence of a potential publication bias was evaluated using funnel plots. Review Manager (version 5.0, The Cochrane Collaboration) was used for all analyses.

\section{RESULTS}

\section{Literature search}

Overall, 685 publications were identified with the electronic search (Figure 1). One additional publication was identified after reviewing the references of relevant published systematic reviews and meta-analyses. ${ }^{14}$ Twenty-nine full-text publications were reviewed, and seven studies were excluded: six used tamsulosin as a therapy combined with a corticosteroid, ${ }^{15-20}$ and one assessed the efficiency of a second cycle of tamsulosin. ${ }^{22}$ Therefore, 22 studies were included, for a total of 1,719 patients.

Studies were written in English ${ }^{14,23-41}$ and Korean..$^{42,43}$ Most articles compared tamsulosin to standard therapy alone (NSAIDs + hydration \pm opioids)..$^{2-29,35-43}$ Seven 


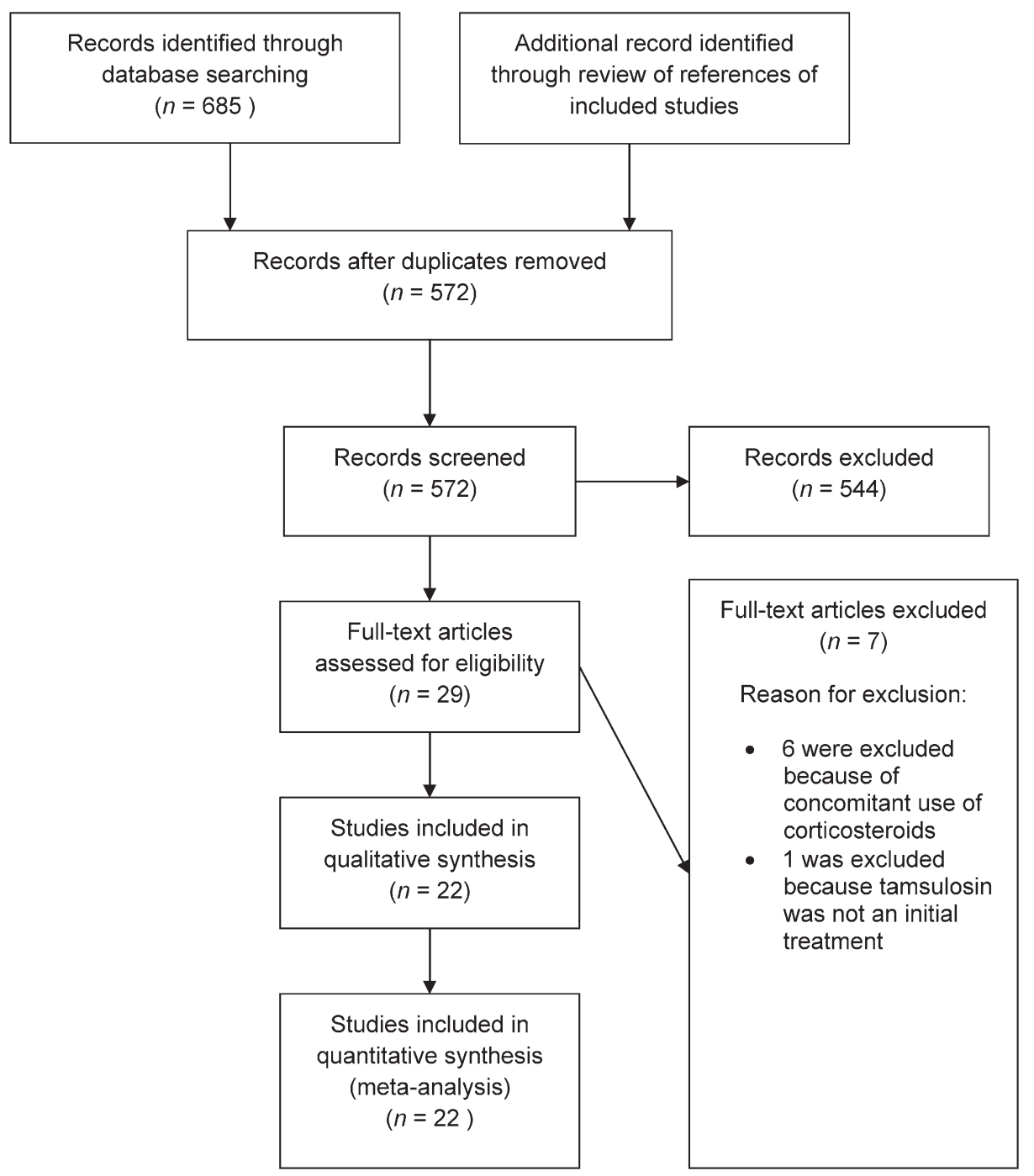

Figure 1. PRISMA flow diagram.

studies administered an additional drug (including antibiotics, a proton pump inhibitor, an antispasmolytic drug, an anticholinergic drug, or an antiexsudative drug) to both groups. ${ }^{14,30-34,36}$ The median time to follow-up after the patient was included in a study was 4 weeks (range 1-8 weeks) (Table 1).

\section{Methodological quality}

Only 4 studies had a low risk of bias, ${ }^{14,27,33,36} 7$ had a moderate risk of bias, ${ }^{24,25,30,32,34,35,38,40}$ and 11 had a high risk of bias ${ }^{23,26,28,29,31,37,39,41-43}$ (Figure 2). A poor description of the randomization process is mainly responsible for the high proportion of moderate or high risk of bias studies. Only four studies were double-blinded, and only six compared tamsulosin to a placebo. We obtained additional information directly from the authors for five studies. ${ }^{24,26,35,36,40}$

\section{Primary outcome: expulsion of ureterolithiasis}

The average size of the distal lithiasis was $\leq 5 \mathrm{~mm}$ in seven studies. Included results suggested a benefit for the expulsion of lithiasis when tamsulosin was combined with a standard treatment (RR 1.50 [95\% CI $1.31-1.71], \mathrm{I}^{2}=70 \%$ ) (Figure 3). All but one study showed a trend toward a favourable association between tamsulosin use and expulsion of lithiasis. Subgroup analysis of low risk of bias studies showed no statistically significant association $(n=4,412$ patients, RR 1.16 [95\% CI 0.92-1.47], $\mathrm{I}^{2}=77 \%$ ) (Table 2). Double-blind randomized studies demonstrated a significant association $(n=5,542$ patients, RR 1.22 [95\% CI 1.06-1.41], $\left.\mathrm{I}^{2}=37 \%\right)$. A subgroup analysis based on the duration of treatment showed little impact on global RR for the expulsion of the lithiasis. Not enough data were available to evaluate the impact 


\begin{tabular}{|c|c|c|c|c|c|c|c|c|}
\hline $\begin{array}{l}\text { Study, } \\
\text { year }\end{array}$ & Comparison & Standard therapy & $\begin{array}{c}\text { Duration of } \\
\text { treatment, } \\
\text { days }\end{array}$ & Intervention & $\begin{array}{c}\text { Participants, } \\
n\end{array}$ & $\begin{array}{l}\text { Mean stone } \\
\text { size, mm } \\
\text { (range) }\end{array}$ & $\begin{array}{l}\text { Stone } \\
\text { expulsion } \\
\text { rate }(\%)\end{array}$ & $\begin{array}{l}\text { Mean time } \\
\text { to } \\
\text { expulsion, } \\
\text { days }\end{array}$ \\
\hline \multirow{2}{*}{$\begin{array}{l}\text { Abdel- } \\
\text { Meguid } \\
\text { et al, } 2010^{24}\end{array}$} & \multirow[t]{2}{*}{$\begin{array}{l}\text { Tamsulosin v. } \\
\text { placebo }\end{array}$} & \multirow[t]{2}{*}{$\begin{array}{l}\text { Hydration, } \\
\text { NSAIDs }\end{array}$} & \multirow[t]{2}{*}{28} & $\begin{array}{l}\text { Tamsulosin } \\
0.4 \mathrm{mg}\end{array}$ & 75 & $(4-10)$ & $61: 75(81)^{* \dagger}$ & NR \\
\hline & & & & Placebo & 75 & $(4-10)$ & $42: 75(56)$ & NR \\
\hline \multirow{2}{*}{$\begin{array}{l}\text { Ahmed and } \\
\text { Al-Sayed, } \\
2010^{25}\end{array}$} & \multirow{2}{*}{$\begin{array}{l}\text { Tamsulosin v. } \\
\text { alfuzosin v. } \\
\text { diclofenac }\end{array}$} & \multirow[t]{2}{*}{$\begin{array}{l}\text { Diclofenac, } \\
\text { tramadol } \mathrm{HCl}\end{array}$} & \multirow[t]{2}{*}{30} & $\begin{array}{l}\text { Tamsulosin } \\
0.4 \mathrm{mg}\end{array}$ & 29 & 4.9 & $25: 29(86)^{* \dagger}$ & 7.5 \\
\hline & & & & Control & 28 & 5.3 & $14: 28(50)$ & 13.9 \\
\hline \multirow{2}{*}{$\begin{array}{l}\text { Agrawal } \\
\text { et al, } \\
2009^{26}\end{array}$} & \multirow{2}{*}{$\begin{array}{l}\text { Tamsulosin v. } \\
\text { alfuzosin v. } \\
\text { placebo }\end{array}$} & \multirow[t]{2}{*}{$\begin{array}{l}\text { Hydration, } \\
\text { diclofenac }\end{array}$} & \multirow[t]{2}{*}{28} & $\begin{array}{l}\text { Tamsulosin } \\
0.4 \mathrm{mg}\end{array}$ & 34 & $6.1(5-9)$ & $28: 34(81)^{* \dagger}$ & 12.3 \\
\hline & & & & Placebo & 34 & $6.3(4-8)$ & $12: 34(36)$ & 24.5 \\
\hline \multirow{2}{*}{$\begin{array}{l}\text { Al-Ansari } \\
\text { et al, } \\
2010^{27}\end{array}$} & \multirow[t]{2}{*}{$\begin{array}{l}\text { Tamsulosin } \\
\text { v. placebo }\end{array}$} & \multirow[t]{2}{*}{$\begin{array}{l}\text { Hydration, } \\
\text { diclofenac }\end{array}$} & \multirow[t]{2}{*}{28} & $\begin{array}{l}\text { Tamsulosin } \\
0.4 \mathrm{mg}\end{array}$ & 50 & 5.8 & $41: 50(82)^{* \dagger}$ & 6.4 \\
\hline & & & & Placebo & 46 & 6.0 & $28: 46(61)$ & 9.8 \\
\hline \multirow{2}{*}{$\begin{array}{l}\text { Aldemir } \\
\text { et al, } \\
2010^{28}\end{array}$} & \multirow{2}{*}{$\begin{array}{l}\text { Tamsulosin v. } \\
\text { Rowatinex v. } \\
\text { diclofenac }\end{array}$} & \multirow[t]{2}{*}{$\begin{array}{l}\text { Hydration, } \\
\text { diclofenac }\end{array}$} & \multirow[t]{2}{*}{10} & $\begin{array}{l}\text { Tamsulosin } \\
0.4 \mathrm{mg}\end{array}$ & 31 & $6.7(4-10)$ & $25: 31(80)^{* \dagger}$ & 3.5 \\
\hline & & & & Control & 29 & $6.6(4-10)$ & $11: 29(37)$ & 7.0 \\
\hline \multirow{2}{*}{$\begin{array}{l}\text { Arrabal- } \\
\text { Martin } \\
\text { et al, } \\
2010^{29}\end{array}$} & \multirow[t]{2}{*}{$\begin{array}{l}\text { Tamsulosin } \\
\text { v. control }\end{array}$} & \multirow{2}{*}{$\begin{array}{l}\text { Hydration, } \\
\text { ibuprofen, } \\
\text { tramadol }\end{array}$} & \multirow[t]{2}{*}{21} & $\begin{array}{l}\text { Tamsulosin } \\
0.4 \mathrm{mg}\end{array}$ & 35 & - & $30: 35(85)^{\ddagger}$ & 8.0 \\
\hline & & & & Control & 35 & - & 19:35 (54) & 13.8 \\
\hline \multirow{2}{*}{$\begin{array}{l}\text { Autorino } \\
\text { et al, } \\
2005^{30}\end{array}$} & \multirow[t]{2}{*}{$\begin{array}{l}\text { Tamsulosin } \\
\text { v. control }\end{array}$} & \multirow{2}{*}{$\begin{array}{l}\text { Hydration, } \\
\text { diclofenac, } \\
\text { aescin, } \\
\text { omeprazole, } \\
\text { levofloxacin }\end{array}$} & \multirow[t]{2}{*}{14} & $\begin{array}{l}\text { Tamsulosin } \\
0.4 \mathrm{mg}\end{array}$ & 32 & $6.5(4-10)$ & $28: 32(88)^{\ddagger}$ & 4.8 \\
\hline & & & & Control & 32 & $5.7(3-10)$ & 19:32 (60) & 7.4 \\
\hline \multirow{2}{*}{$\begin{array}{l}\text { Avdoshin } \\
\text { et al, } \\
2005^{31}\end{array}$} & $\begin{array}{l}\text { Tamsulosin } \\
\text { v. control }\end{array}$ & Anticholinergic & - & $\begin{array}{l}\text { Tamsulosin } \\
0.4 \mathrm{mg}\end{array}$ & 42 & - & $31: 42(74)^{\ddagger}$ & NR \\
\hline & & & & Control & 45 & - & $11: 45(24)$ & NR \\
\hline $\begin{array}{l}\text { Cervenakov } \\
\text { et al, }\end{array}$ & $\begin{array}{l}\text { Tamsulosin } \\
\text { v. control }\end{array}$ & $\begin{array}{l}\text { Hydration, } \\
\text { Tramal, }\end{array}$ & 7 & $\begin{array}{l}\text { Tamsulosin } \\
0.4 \mathrm{mg}\end{array}$ & 51 & $(1-10)$ & $41: 51(80)^{*^{\dagger}}$ & 3.1 \\
\hline $2002^{32}$ & & $\begin{array}{l}\text { diazepam, } \\
\text { Yellon, diclofenac }\end{array}$ & & Control & 51 & $(1-10)$ & $32: 51$ (63) & 3.4 \\
\hline $\begin{array}{l}\text { De Sio } \\
\text { et al, }\end{array}$ & $\begin{array}{l}\text { Tamsulosin } \\
\text { v. control }\end{array}$ & $\begin{array}{l}\text { Hydration, } \\
\text { diclofenac, }\end{array}$ & 14 & $\begin{array}{l}\text { Tamsulosin } \\
0.4 \mathrm{mg}\end{array}$ & 46 & 6.9 & $45: 50(90)^{\ddagger}$ & 4.4 \\
\hline $2006^{33}$ & & $\begin{array}{l}\text { aescin, } \\
\text { omeprazole, } \\
\text { levofloxacin }\end{array}$ & & Control & 50 & 6.4 & $27: 46(58)$ & 7.5 \\
\hline $\begin{array}{l}\text { Erturhan } \\
\text { et al, }\end{array}$ & $\begin{array}{l}\text { Tamsulosin v. } \\
\text { tamsulosin }+\end{array}$ & $\begin{array}{l}\text { Hydration, } \\
\text { diclofenac, }\end{array}$ & 21 & $\begin{array}{l}\text { Tamsulosin } \\
0.4 \mathrm{mg}\end{array}$ & 30 & 7.1 & $22: 30(73)^{* \dagger}$ & 6.4 \\
\hline $2007^{34}$ & $\begin{array}{l}\text { tolterodine } \mathrm{v} \text {. } \\
\text { tolterodine } \\
\mathrm{v} \text {. control }\end{array}$ & cefuroxime & & Control & 30 & 6.8 & $12: 30(40)$ & 12.2 \\
\hline $\begin{array}{l}\text { Ferre } \\
\text { et al, }\end{array}$ & $\begin{array}{l}\text { Tamsulosin } \\
\text { v. control }\end{array}$ & $\begin{array}{l}\text { Ibuprofen, } \\
\text { oxycodone }\end{array}$ & 10 & $\begin{array}{l}\text { Tamsulosin } \\
0.4 \mathrm{mg}\end{array}$ & 38 & 3.5 & $27: 38(71)^{*}$ & 2.6 \\
\hline $2009^{35}$ & & & & Control & 39 & 3.8 & $24: 39(61)$ & 3.2 \\
\hline $\begin{array}{l}\text { Han et al, } \\
2006^{42}\end{array}$ & $\begin{array}{l}\text { Tamsulosin } \\
\text { v. carvoverine }\end{array}$ & Ketorolac & 28 & $\begin{array}{l}\text { Tamsulosin } \\
0.2 \mathrm{mg}\end{array}$ & 35 & 4.4 & 29:35 (82) & 4.6 \\
\hline & & & & Carvoverine & 32 & 4.3 & $17: 32(53)$ & 8.3 \\
\hline $\begin{array}{l}\text { Hermanns } \\
\text { et al, }\end{array}$ & $\begin{array}{l}\text { Tamsulosin } \\
\text { v. placebo }\end{array}$ & $\begin{array}{l}\text { Diclofenac, } \\
\text { metamizole }\end{array}$ & 21 & $\begin{array}{l}\text { Tamsulosin } \\
0.4 \mathrm{mg}\end{array}$ & 45 & $4.1(3-5)$ & $39: 45(86)^{\dagger}$ & NR \\
\hline $2009^{36}$ & & & & Placebo & 45 & $3.8(3-5)$ & 40:45 (88) & $N R$ \\
\hline $\begin{array}{l}\text { Kim et al, } \\
2007^{43}\end{array}$ & $\begin{array}{l}\text { Tamsulosin } \\
\text { v. control }\end{array}$ & $\begin{array}{l}\text { Hydration, } \\
\text { ketorolac }\end{array}$ & 28 & $\begin{array}{l}\text { Tamsulosin } \\
\text { 0/2 mg }\end{array}$ & 21 & 4.8 & $19: 21(90)$ & 12.2 \\
\hline & & & & Control & 20 & 5.9 & $12: 20(60)$ & 17.3 \\
\hline
\end{tabular}




\begin{tabular}{|c|c|c|c|c|c|c|c|c|}
\hline $\begin{array}{l}\text { Study, } \\
\text { year }\end{array}$ & Comparison & Standard therapy & $\begin{array}{l}\text { Duration of } \\
\text { treatment, } \\
\text { days }\end{array}$ & Intervention & $\begin{array}{c}\text { Participants, } \\
n\end{array}$ & $\begin{array}{l}\text { Mean stone } \\
\text { size, mm } \\
\text { (range) }\end{array}$ & $\begin{array}{l}\text { Stone } \\
\text { expulsion } \\
\text { rate (\%) }\end{array}$ & $\begin{array}{l}\text { Mean time } \\
\text { to } \\
\text { expulsion, } \\
\text { days }\end{array}$ \\
\hline \multirow{2}{*}{$\begin{array}{l}\text { Küpeli } \\
\text { et al, } \\
2004^{37}\end{array}$} & \multirow[t]{2}{*}{$\begin{array}{l}\text { Tamsulosin } \\
\text { v. control }\end{array}$} & \multirow[t]{2}{*}{$\begin{array}{l}\text { Hydration, } \\
\text { diclofenac }\end{array}$} & \multirow[t]{2}{*}{15} & $\begin{array}{l}\text { Tamsulosin } \\
0.4 \mathrm{mg}\end{array}$ & 15 & $4.7(3-5)$ & $8: 15(53)^{\dagger}$ & NR \\
\hline & & & & Control & 15 & $4.9(3-5)$ & $3: 15(20)$ & NR \\
\hline \multirow{3}{*}{$\begin{array}{l}\text { Lojanapiwat } \\
\text { et al, } \\
2008^{38}\end{array}$} & \multirow{3}{*}{$\begin{array}{l}\text { Tamsulosin } \\
0.2 \text { mg v. } \\
\text { tamsulosin } \\
0.4 \text { mg } \\
\text { v. control }\end{array}$} & \multirow[t]{3}{*}{ Diclofenac } & \multirow[t]{3}{*}{28} & $\begin{array}{l}\text { Tamsulosin } \\
0.2 \mathrm{mg}\end{array}$ & 25 & $6.4(4-10)$ & $10: 25(40)^{*}$ & 9.3 \\
\hline & & & & $\begin{array}{l}\text { Tamsulosin } \\
0.4 \mathrm{mg}\end{array}$ & 25 & $6.2(4-10)$ & $17: 25(68)$ & 10.8 \\
\hline & & & & Control & 25 & $6.7(4-10)$ & $1: 25(4)$ & 23.0 \\
\hline \multirow{2}{*}{$\begin{array}{l}\text { Ochoa- } \\
\text { Gomez } \\
\text { et al, } \\
2011^{23}\end{array}$} & \multirow[t]{2}{*}{$\begin{array}{l}\text { Tamsulosin } \\
\text { v. placebo }\end{array}$} & \multirow{2}{*}{$\begin{array}{l}\text { Hydration, } \\
\text { conventional } \\
\text { therapy } \\
\text { unspecified }\end{array}$} & \multirow[t]{2}{*}{28} & $\begin{array}{l}\text { Tamsulosin } \\
0.4 \mathrm{mg}\end{array}$ & 32 & 5.3 & $22: 32(69)^{\dagger}$ & 22.0 \\
\hline & & & & Placebo & 33 & 5.2 & $23: 33(70)$ & 23.0 \\
\hline \multirow{2}{*}{$\begin{array}{l}\text { Sayed } \\
\text { et al, } \\
2008^{39}\end{array}$} & \multirow[t]{2}{*}{$\begin{array}{l}\text { Tamsulosin } \\
\text { v. control }\end{array}$} & \multirow[t]{2}{*}{$\begin{array}{l}\text { Hydration, } \\
\text { diclofenac }\end{array}$} & \multirow[t]{2}{*}{28} & $\begin{array}{l}\text { Tamsulosin } \\
0.4 \mathrm{mg}\end{array}$ & 45 & $6.8(5-10)$ & $40: 45(88)^{\dagger}$ & 7.3 \\
\hline & & & & Control & 45 & $6.4(5-10)$ & $23: 45(51)$ & 12.5 \\
\hline \multirow{2}{*}{$\begin{array}{l}\text { Vincendeau } \\
\text { et al, } \\
2010^{13}\end{array}$} & \multirow[t]{2}{*}{$\begin{array}{l}\text { Tamsulosin } \\
\text { v. placebo }\end{array}$} & \multirow[t]{2}{*}{$\begin{array}{l}\text { Ketoprofen, } \\
\text { phloroglucinol }\end{array}$} & \multirow[t]{2}{*}{42} & $\begin{array}{l}\text { Tamsulosin } \\
0.4 \mathrm{mg}\end{array}$ & 66 & $2.9(2-7)$ & $47: 66(77)^{* \dagger}$ & 9.6 \\
\hline & & & & Placebo & 63 & $3.2(2-7)$ & $43: 63(70)$ & 10.1 \\
\hline \multirow[t]{2}{*}{$\begin{array}{l}\text { Wang et al, } \\
2008^{40}\end{array}$} & \multirow[t]{2}{*}{$\begin{array}{l}\text { Tamsulosin } \\
\text { v. control }\end{array}$} & \multirow{2}{*}{$\begin{array}{l}\text { Hydration, } \\
\text { ketorolac, } \\
\text { buprenorphine }\end{array}$} & \multirow[t]{2}{*}{14} & $\begin{array}{l}\text { Tamsulosin } \\
0.4 \mathrm{mg}\end{array}$ & 32 & $6.5(5-9)$ & $26: 32(81)^{\dagger}$ & 6.3 \\
\hline & & & & Control & 31 & $6.5(4-9)$ & $17: 31(55)$ & 10.1 \\
\hline \multirow[t]{2}{*}{$\begin{array}{l}\text { Yilmaz et al, } \\
2005^{41}\end{array}$} & \multirow{2}{*}{$\begin{array}{l}\text { Tamsulosin } \\
\text { v. terazosin } \\
\text { v. doxazosin } \\
\text { v. control }\end{array}$} & \multirow[t]{2}{*}{$\begin{array}{l}\text { Hydration, } \\
\text { diclofenac }\end{array}$} & \multirow[t]{2}{*}{28} & $\begin{array}{l}\text { Tamsulosin } \\
0.4 \mathrm{mg}\end{array}$ & 29 & 6.0 & $23: 29(79)^{*^{\dagger}}$ & 6.3 \\
\hline & & & & Control & 28 & 6.0 & $15: 28(53)$ & 10.5 \\
\hline
\end{tabular}

of dosage on the stone expulsion rate. Subgroup analyses for American and European studies are represented in Figure 4A $(n=12,900$ patients, RR 1.33 [95\% CI 1.14-1.1.55], $\mathrm{I}^{2}=66 \%$ ), and subgroup analyses for Asian, Arabic, and Russian studies are represented in Figure 4B $(n=11,819$ patients, RR 1.71 [95\% CI 1.43-2.03], $\mathrm{I}^{2}=51 \%$ ).

Ten studies had a mean stone size $>5 \mathrm{~mm}$. This subgroup demonstrated a significant benefit for the spontaneous expulsion rate with tamsulosin $(n=10,754$ patients, RR 1.65 [95\% CI 1.42-1.93], $\mathrm{I}^{2}=37 \%$ ). Tamsulosin showed a smaller benefit for the eight studies with a mean stone size $\leq 5 \mathrm{~mm}(n=550$, RR 1.22 [95\% CI 1.02-1.47], $\left.\mathrm{I}^{2}=62 \%\right)$. Subgroup analysis of RCTs published since the last meta-analysis also demonstrated a smaller but still significant benefit for the expulsion rate with tamsulosin $(n=10,862$ patients, RR 1.32 [95\% CI 1.12-1.61], $\mathrm{I}^{2}=75 \%$ ) (Figure 5). The funnel plot visually demonstrates a moderate asymmetry (Figure 6).

\section{Secondary outcomes}

Eighteen studies evaluated the mean time to distal lithiasis expulsion. Among these, 13 reported standard deviations and could be included in the meta-analysis (Figure 7). Tamsulosin was associated with a decrease of 3.33 days in the mean time to stone expulsion (95\% CI $\left.-4.23,-2.44, \mathrm{I}^{2}=67 \%\right)$. Sensitivity analyses showed consistent findings (Table 3). Five studies reported the impact of tamsulosin on the number of hospitalizations or medical consultations due to recurrent colics, but no significant association could be found ( $n=5,400$ patients, RR 0.57 [95\% CI 0.31 $\left.1.03], \mathrm{I}^{2}=0 \%\right)$. Five studies (353 patients) reported the average number of pain episodes; however, no clinically significant benefit could be shown with a meta-analysis.

Only 16 studies reported side effects. Overall, 8 patients receiving tamsulosin reported retrograde ejaculation $(p<0.01), 29$ complained of gastrointestinal 


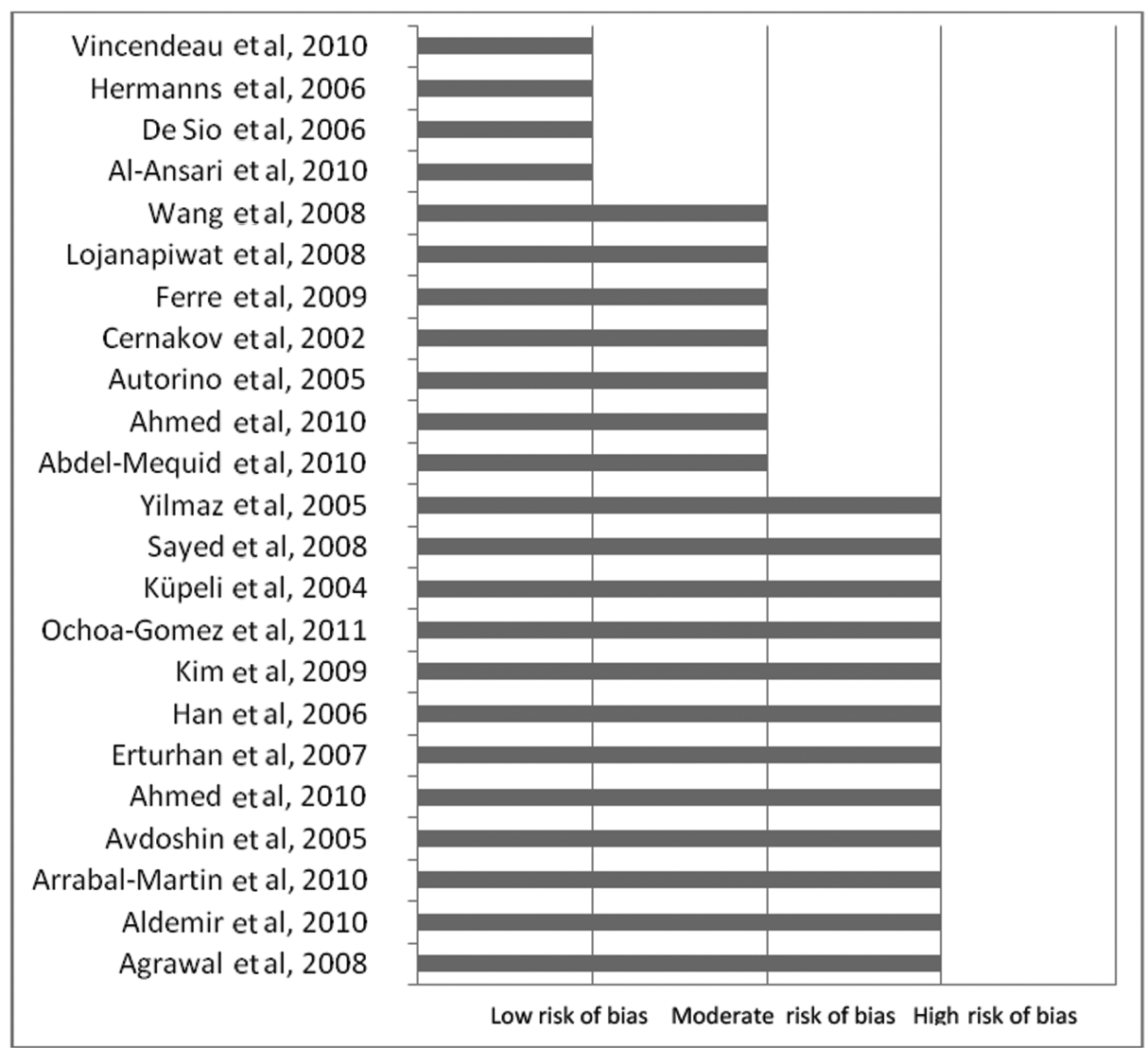

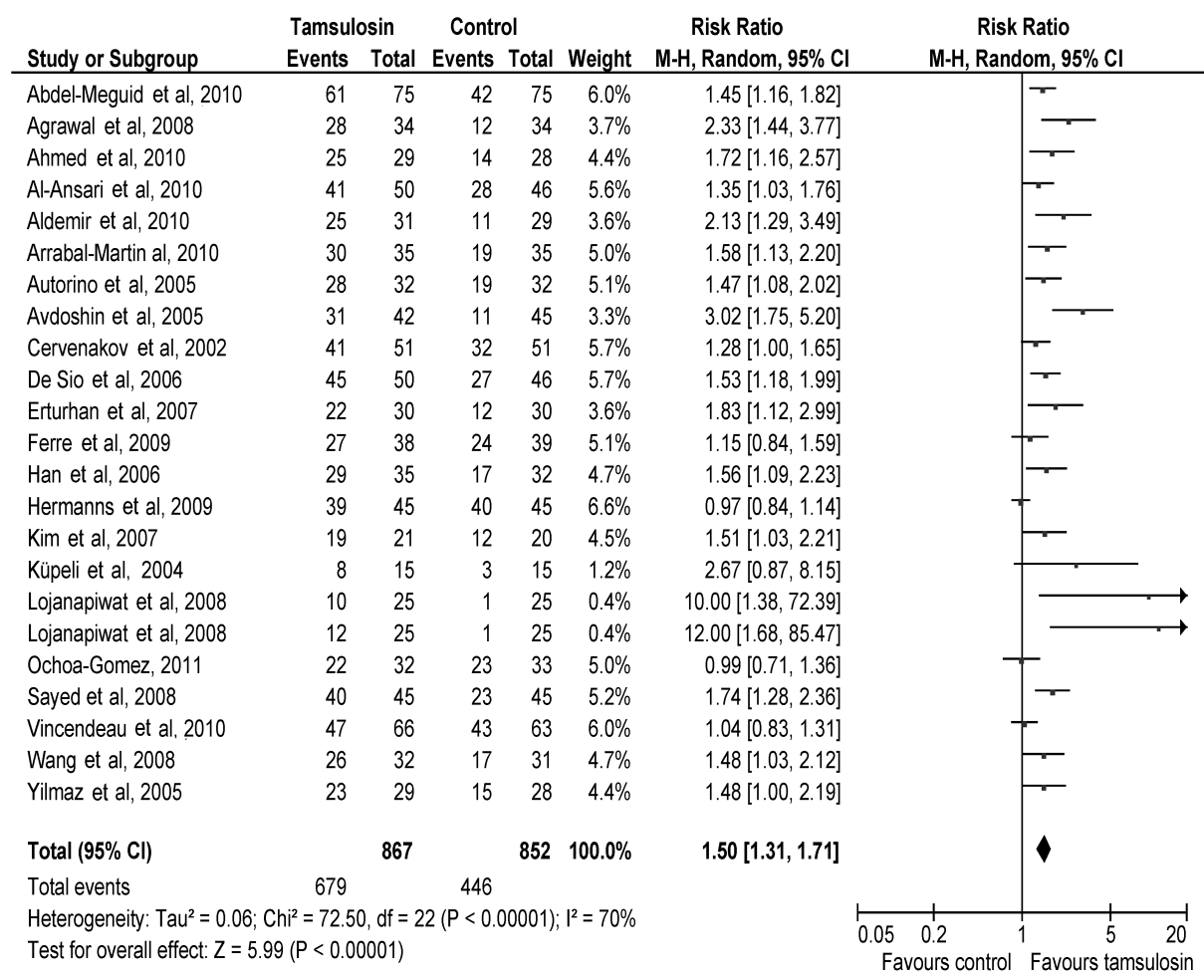

Figure 2. Assessment of the quality of included studies using the Cochrane risk of bias assessment tool.

Figure 3. Forest plot for the stone expulsion rate for all included studies: tamsulosin versus control. $\mathrm{M}-\mathrm{H}=$ Mantel-Haenszel. 


\begin{tabular}{|c|c|c|c|c|}
\hline Sensitivity or subgroup analysis & Study $(n)$ & Participants $(n)$ & Risk ratio $(95 \% \mathrm{Cl})^{*}$ & $1^{2}$ \\
\hline Spontaneous expulsion rate for double-blind studies & 5 & 542 & $1.22(1.06,1.41)$ & 37 \\
\hline Spontaneous expulsion rate for low risk of bias studies & 4 & 412 & $1.16(0.92,1.47)$ & 77 \\
\hline $\begin{array}{l}\text { Spontaneous expulsion rate for studies with mean stone size } \\
\leq 5 \mathrm{~mm}\end{array}$ & 8 & 550 & $1.22(1.02,1.47)$ & 62 \\
\hline $\begin{array}{l}\text { Spontaneous expulsion rate for studies with mean stone size } \\
>5 \mathrm{~mm} \text { but } \leq 10 \mathrm{~mm}\end{array}$ & 10 & 754 & $1.65(1.42,1.93)$ & 37 \\
\hline Spontaneous expulsion rate for treatment $<21$ days & 7 & 492 & $1.43(1.25,1.64)$ & 9 \\
\hline Spontaneous expulsion rate for treatment $\geq 21$ days & 14 & 1140 & $1.47(1.23,1.71)$ & 75 \\
\hline
\end{tabular}

symptoms $(p=0.20), 11$ complained of orthostatic hypotension $(p=0.08), 9$ complained of dizziness $(p=0.30)$, and 14 suffered from headache $(p=0.37), 27$ from asthenia $(p=0.36), 3$ from rhinitis $(p=0.62)$, and 3 from palpitations $(p=0.62)$. No study has documented patients' compliance with the treatment.

\section{DISCUSSION}

We observed a significant effect of tamsulosin in improving the rate of spontaneous expulsion of distal ureterolithiasis $\leq 10 \mathrm{~mm}$. This benefit was observed to be potentially larger with a mean ureterolithiasis size $>5 \mathrm{~mm}$ but still significantly beneficial for smaller
A For North American/South American and European studies

\begin{tabular}{|c|c|c|c|c|c|c|}
\hline \multirow[b]{2}{*}{ Study or Subgroup } & \multicolumn{2}{|c|}{ Tamsulosin } & \multicolumn{2}{|c|}{ Control } & \multirow[b]{2}{*}{ Weight } & \\
\hline & Events & Total & Events & Total & & $\mathrm{M}-\mathrm{H}$ \\
\hline Aldemir et al. 2010 & 25 & 31 & 11 & 29 & $5.8 \%$ & \\
\hline Arrabal-Martin al, 2010 & 30 & 35 & 19 & 35 & $8.6 \%$ & \\
\hline Autorino et al, 2005 & 28 & 32 & 19 & 32 & $9.0 \%$ & \\
\hline Cervenakov et al, 2002 & 41 & 51 & 32 & 51 & $10.4 \%$ & \\
\hline De Sio et al, 2006 & 45 & 50 & 27 & 46 & $10.2 \%$ & \\
\hline Erturhan et al, 2007 & 22 & 30 & 12 & 30 & $5.9 \%$ & \\
\hline Ferre et al, 2009 & 27 & 38 & 24 & 39 & $8.9 \%$ & \\
\hline Hermanns et al, 2009 & 39 & 45 & 40 & 45 & $12.5 \%$ & \\
\hline Küpeli et al, 2004 & 8 & 15 & 3 & 15 & $1.7 \%$ & \\
\hline Ochoa-Gomez, 2011 & 22 & 32 & 23 & 33 & $8.8 \%$ & \\
\hline Vincendeau et al, 2010 & 47 & 66 & 43 & 63 & $10.9 \%$ & \\
\hline Yilmaz et al, 2005 & 23 & 29 & 15 & 28 & $7.5 \%$ & \\
\hline Total $(95 \% \mathrm{Cl})$ & & 454 & & 446 & $100.0 \%$ & \\
\hline Total events & 357 & & 268 & & & \\
\hline Heterogeneity: $\operatorname{Tau}^{2}=0$. & $\mathrm{Chi}^{2}=31$ & $96, d f=$ & $11(P=0$ & $0.0008)$ & $; 1^{2}=66 \%$ & \\
\hline Test for overall effect: $Z$ & $.57(P=0$. & 0004) & & & & \\
\hline
\end{tabular}

Risk Ratio

$\mathrm{H}$, Random, $95 \% \mathrm{Cl}$

$2.13[1.29,3.49]$

$1.58[1.13,2.20]$

$1.47[1.08,2.02]$

$1.28[1.00,1.65]$

$1.53[1.18,1.99]$

$1.83[1.12,2.99]$

$1.15[0.84,1.59]$

$0.97[0.84,1.14]$

$2.67[0.87,8.15]$

$0.99[0.71,1.36]$

$1.04[0.83,1.31]$

$1.48[1.00,2.19]$

$1.33[1.14,1.55]$

$\mathrm{M}-\mathrm{H}$, Random, $95 \% \mathrm{Cl}$

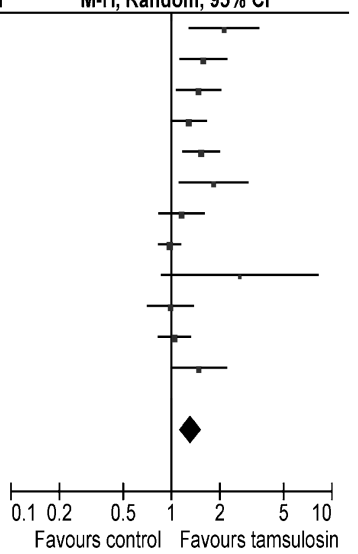

B For Asian, Russian, Egyptian, and Arabian studies

\begin{tabular}{|c|c|c|c|c|c|c|c|c|}
\hline \multirow{2}{*}{$\begin{array}{l}\text { Study or Subgroup } \\
\text { Abdel-Meguid et al, } 2010\end{array}$} & \multicolumn{2}{|c|}{ Tamsulosin } & Control & rol & Weight & $\begin{array}{c}\text { Risk Ratio } \\
\text { M-H, Random, } 95 \% \mathrm{Cl}\end{array}$ & \multicolumn{2}{|c|}{$\begin{array}{c}\text { Risk Ratio } \\
\text { M-H, Random, } 95 \% \mathrm{Cl}\end{array}$} \\
\hline & 61 & 75 & 42 & 75 & $15.1 \%$ & $1.45[1.16,1.82]$ & & $\rightarrow$ \\
\hline Agrawal et al, 2008 & 28 & 34 & 12 & 34 & $8.0 \%$ & $2.33[1.44,3.77]$ & & \\
\hline Ahmed et al, 2010 & 25 & 29 & 14 & 28 & $9.9 \%$ & $1.72[1.16,2.57]$ & & $\longrightarrow$ \\
\hline Al-Ansari et al, 2010 & 41 & 50 & 28 & 46 & $13.8 \%$ & $1.35[1.03,1.76]$ & & \\
\hline Avdoshin et al, 2005 & 31 & 42 & 11 & 45 & $6.9 \%$ & $3.02[1.75,5.20]$ & & \\
\hline Han et al, 2006 & 29 & 35 & 17 & 32 & $11.0 \%$ & $1.56[1.09,2.23]$ & & \\
\hline Kim et al, 2007 & 19 & 21 & 12 & 20 & $10.3 \%$ & $1.51[1.03,2.21]$ & & \\
\hline Lojanapiwat et al, 2008 & 12 & 25 & 1 & 25 & $0.8 \%$ & $12.00[1.68,85.47]$ & & \\
\hline Lojanapiwat et al, 2008 & 10 & 25 & 1 & 25 & $0.8 \%$ & $10.00[1.38,72.39]$ & & \\
\hline Sayed et al, 2008 & 40 & 45 & 23 & 45 & $12.6 \%$ & $1.74[1.28,2.36]$ & & 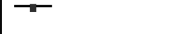 \\
\hline Wang et al, 2008 & 26 & 32 & 17 & 31 & $10.9 \%$ & $1.48[1.03,2.12]$ & & $\longrightarrow$ \\
\hline Total $(95 \% \mathrm{Cl})$ & & 413 & & 406 & $100.0 \%$ & $1.71[1.43,2.03]$ & & \\
\hline Total events & 322 & & 178 & & & & & \\
\hline $\begin{array}{l}\text { Heterogeneity: } \text { Tau }^{2}=0.04 \\
\text { Test for overall effect: } Z=\end{array}$ & $\begin{array}{l}h i^{2}=20.53 \\
9(P<0.00\end{array}$ & $\begin{array}{l}3, d f=1 \\
0001)\end{array}$ & $0(P=0.0$ & $02) ; I^{2}=$ & $=51 \%$ & & 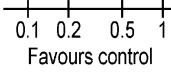 & $\begin{array}{cccc}1 & 2 & 5 & 10 \\
\text { Favours tamsulosin }\end{array}$ \\
\hline
\end{tabular}

Figure 4. Forest plot for the stone expulsion rate: tamsulosin versus control. Subgroup analysis by country of origin. $\mathrm{M}-\mathrm{H}=$ Mantel-Haenszel. 


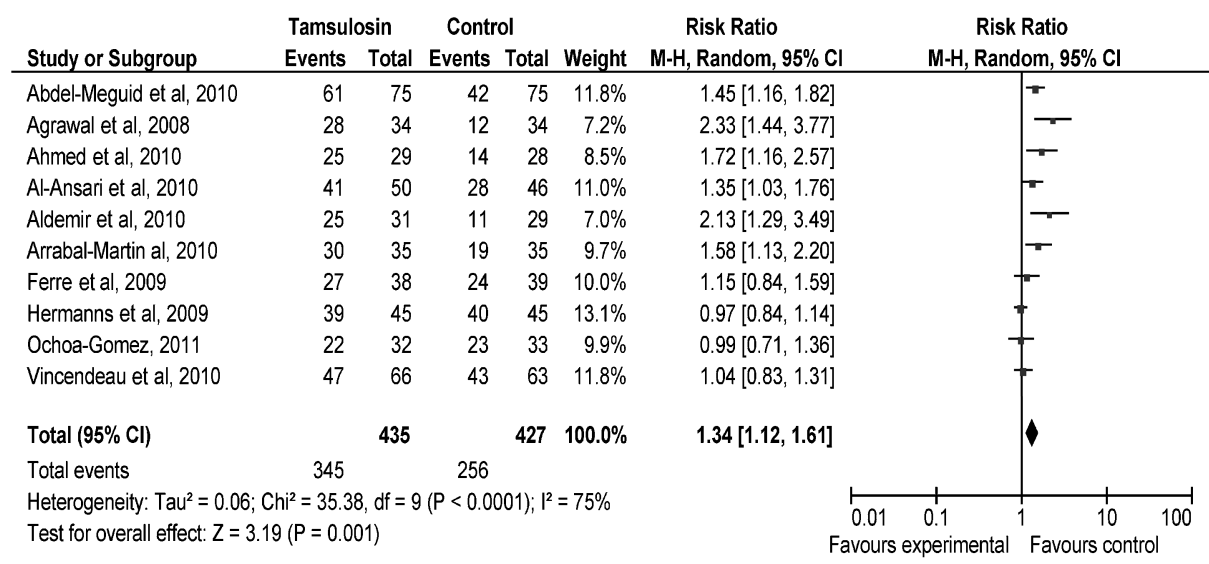

Figure 5. Forest plot for the stone expulsion rate for randomized controlled trials published since the last meta-analysis: tamsulosin versus control. $\mathrm{M}-\mathrm{H}=$ MantelHaenszel. distal ureterolithiasis. We observed a 3-day reduction in the mean time to ureterolithiasis expulsion, but the restricted analysis to low-bias studies showed no gain in the mean time to expulsion with tamsulosin. The stratification by countries of the included studies demonstrated a significant RR just slightly lower for the spontaneous expulsion rate in the American/ European studies, where most likely these patients were managed by emergency physicians, suggesting that the treatment is appropriate for ED use. The main significant side effects associated with tamsulosin use were gastrointestinal symptoms and retrograde ejaculation; however, overall, tamsulosin had a good sideeffect profile.

Our study showed results consistent with those of four previous meta-analyses ${ }^{8-11}$ but provided new insight from low-bias studies results. Since the last published meta-analysis, we found and included 10

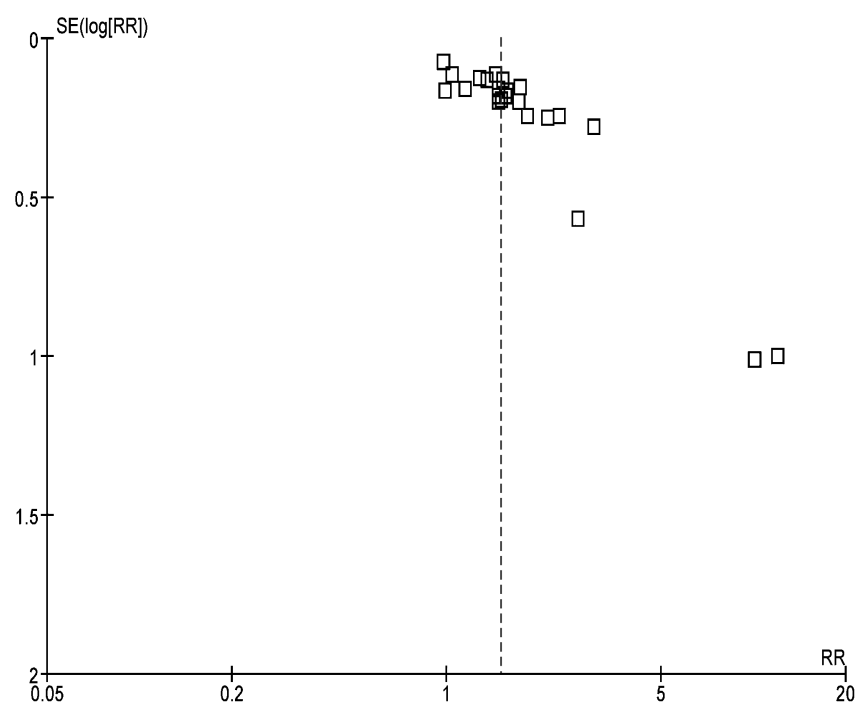

Figure 6. Funnel plots for all included studies over primary outcome (expulsion of lithiasis). additional studies. ${ }^{14,21-27,33,35}$ To compare these previous meta-analyses and evaluate differences, our study reported the results in two ways: the 10 most recent studies since the last meta-analysis and the combined results. ${ }^{8}$ Our study focused solely on tamsulosin for distal ureterolithiasis and not all $\alpha$-antagonists, as previously published by Seitz and colleagues, Hollingsworth and colleagues, Singh and colleagues, and Parsons and colleagues. ${ }^{8-11}$ Distal ureterolithiasis is a situation frequently encountered by emergency physicians and relevant to their clinical practice. ${ }^{11}$ Specialized urologic care is usually needed for large $(>10 \mathrm{~mm})$ and proximal ureterolithiasis. We found that tamsulosin seems to significantly increase the expulsion rate for stones $>5 \mathrm{~mm}$, which is consistent with the only previous meta-analysis that explored this issue. ${ }^{8}$ Our results add evidence that mean stone size has a significant impact on tamsulosin's effect on the rate of spontaneous expulsion.

We also found that stone size had an impact on tamsulosin's efficacy of reducing the mean time to stone expulsion. Tamsulosin was more effective in shortening the time to stone expulsion for a mean stone size $>5 \mathrm{~mm}$. None of the previous systematic reviews evaluated that issue, which is clinically important in renal colic management. A gain of 3 days in expulsion could be of great benefit because most ED renal colic patients tend to be young active patients, with significant costs attributed to lost earnings associated with this medical condition. ${ }^{3}$ Our study also evaluated the impact of tamsulosin and stone size on the mean time to stone expulsion, which had not been evaluated in previous systematic reviews. Our analysis restricted to low-bias studies showed no significant gain in expulsion when exposed to tamsulosin, but heterogeneity was important $\left(\mathrm{I}^{2}=77 \%\right)$. 


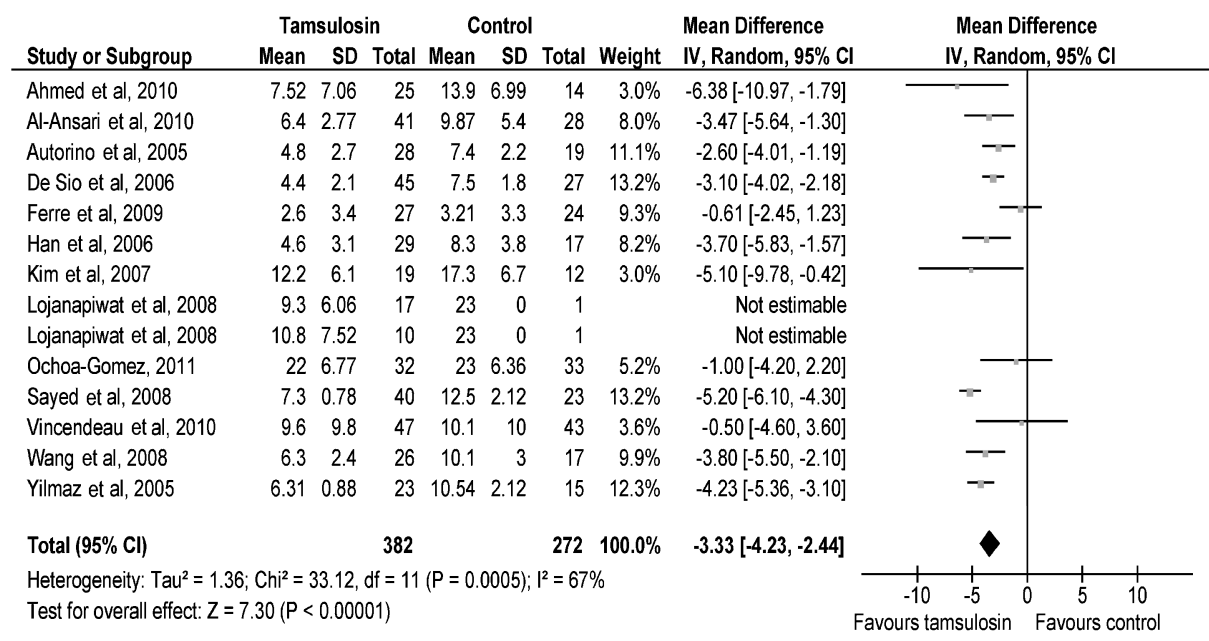

Figure 7. Forest plot for the mean time difference to stone expulsion.
Recently, an updated version of guidelines for the treatment of ureterolithiasis developed by the European Association of Urology (EAU) and the American Urological Association (AUA) reported that $\alpha$-blockers (such as tamsulosin) may facilitate the spontaneous passage of ureterolithiasis $<10 \mathrm{~mm}^{44}$ and that this treatment could reduce the need for analgesia (level of evidence 1A). Our study did not show a significant reduction in the average number of pain episodes. A trend toward a reduction in the number of secondary hospitalizations due to recurrent colic was observed. The EAU and AUA also came to the conclusion that ureterolithiasis $<5 \mathrm{~mm}$ was more often expulsed spontaneously and that treatment with an $\alpha$-blocker may be less likely to be beneficial (level of evidence 1B). We observed comparable results, although our analysis was based on the mean size of ureterolithiasis as an inclusion criterion in studies rather than on the actual size of ureterolithiasis within studies.

\section{Strengths and limitations}

Our systematic review with meta-analysis had numerous strengths. We followed high methodological standards $^{45}$ and conducted a comprehensive literature search. Study selection was done in duplicate, and the methodological quality of included studies was assessed using a recently published standard (Cochrane risk of bias assessment tool) that allowed restricted analyses to low-bias studies. We also searched and found additional information by contacting the corresponding authors. We excluded studies that used a combination of tamsulosin and corticosteroids because some evidence suggests that corticosteroids increase the spontaneous passage of distal ureterolithiasis. ${ }^{15-20}$ Furthermore, our study focused on tamsulosin because this drug seems to have the best safety profile and is the most prescribed $\alpha$ blocker in the ED. Application of results to a similar clinical practice is a pragmatic question before considering a new therapy in the ED. Our meta-analysis

Table 3. Mean time for stone expulsion: tamsulosin versus control or placebo

\begin{tabular}{|c|c|c|c|c|}
\hline Outcome or subgroup & Study $(n)$ & Participants $(n)$ & Mean difference $(\mathrm{IV}, 95 \% \mathrm{Cl})^{*}$ & $\mathrm{I}^{2}$ \\
\hline Mean time for stone expulsion for low risk of bias studies & 3 & 282 & $-3.08(-3.79,-2.38)$ & 0 \\
\hline Mean time for stone expulsion in double-blind studies & 3 & 224 & $-2.18(-4.09,-0.28)$ & 20 \\
\hline $\begin{array}{l}\text { Mean time for stone expulsion for studies with mean stone } \\
\text { size } \leq 5 \mathrm{~mm}\end{array}$ & 7 & 391 & $-2.66(-4.19,-1.13)$ & 50 \\
\hline $\begin{array}{l}\text { Mean time for stone expulsion for studies with mean stone } \\
\text { size }>5 \text { but } \leq 10 \mathrm{~mm}\end{array}$ & 6 & 332 & $-3.80(-4.69,-2.92)$ & 66 \\
\hline Mean time for stone expulsion for treatment $<21$ days & 4 & 213 & $-2.37(-3.88,-0.86)$ & 80 \\
\hline Mean time for stone expulsion for treatment $\geq 21$ days & 8 & 441 & $-3.99(-5.01,-2.97)$ & 46 \\
\hline $\begin{array}{l}\text { Mean time for stone expulsion for RCTs published since } \\
\text { last meta-analysis }\end{array}$ & 5 & 314 & $-2.10(-3.97,-0.23)$ & 52 \\
\hline $\begin{array}{l}\text { IV = inverse variance. } \\
\text { RCT = randomized controlled trial. } \\
{ }^{*} \text { Mantel-Haenszel, random. }\end{array}$ & & & & \\
\hline
\end{tabular}


provided key answers by reporting stratified analyses by country of the included studies.

Our study is limited by the low methodological quality of the included studies. In addition, we cannot exclude the presence of a potential publication bias based on the visual evaluation of the funnel plot for the primary outcome. There is a theoretical risk that negative studies were underrepresented. A publication bias might have affected the results of our study and led to potential overestimation of the summary estimates. Statistical heterogeneity was also observed between included studies. Although it is not unusual in meta-analyses, our planned sensitivity analyses could not explain all the heterogeneity. The rate of spontaneous expulsion of ureterolithiasis in control groups, the different substances administered in control groups, the number of radiologic procedures used to diagnose ureterolithiasis, the various techniques used to measure them, the different definitions of distal lithiasis, and the variation in the length of follow-ups might also explain part of the observed heterogeneity. The assessment of main outcome and the expulsion of stone were quite variable, ranging from self-report by the patient to CT scan follow-up. Finally, we cannot exclude the possibility that the incidence of side effects may have been underestimated considering that no study systematically searched for them.

\section{CONCLUSION}

We observed that tamsulosin was efficient in increasing the rate of successful spontaneous passage of distal ureterolithiasis $\leq 10 \mathrm{~mm}$ consistent across all participating countries, with a good side-effect profile. The mean time to expulsion was significantly reduced in all trials, but this benefit was not reproduced when only the low-bias trials were included. Because most renal colic encountered by emergency physicians is caused by small distal ureterolithiasis $(\leq 10 \mathrm{~mm})$, the results of this study suggest that emergency physicians should consider routine treatment with tamsulosin for these patients. Any future lowbias trials may want to address the time to expulsion inconsistency across studies and should consider stratifying for size. In addition, future studies should clearly record side effects, the randomization process, and pain measurement and should have adequate power to determine a clinically significant benefit in expulsion.

Acknowledgements: We would like to thank Ms. Valérie Boucher for translation and review and Ms. Amélie Boutin for review of this article.
Competing interests: Dr. Turgeon is supported by a Research Career Award from the Fonds de la Recherche en Santé du Québec.

\section{REFERENCES}

1. Stamatelou KK, Franci ME, Jones CA, et al. Time trends in reported prevalence of kidney stones in the United States: 1976-1994. Kidney Int 2003;63:1817, doi:10.1046/j.15231755.2003.00917.x.

2. Marx J. Rosen's emergency medicine. Vol 1. 7th ed. Philadelphia: Elsevier; 2010.

3. Pearle MS, Calhoun EA, Curhan GC. Urologic Diseases in America Project: urolithiasis. 7 Urol 2005;173:848-57, doi: 10.1097/01.ju.0000152082.14384.d7.

4. Coll DM, Varanelli MJ, Smith RC. Relationship of spontaneous passage of ureteral calculi to stone size and location as revealed by unenhanced helical CT. A7R Am 7 Roentgenol 2002;178:101-3, doi:10.2214/ajr.178.1.1780101.

5. Türk C, Knoll T, Petrik A, et al. Guidelines on urolithiasis European Association of Urology 2010. Available at: http:// www.uroweb.org (accessed November 10, 2011).

6. Chandhoke PS. When is medical prophylaxis cost-effective for recurrent calcium stones? $f$ Urol 2002;168:937-40, doi:10.1016/S0022-5347(05)64546-6.

7. Daniels GJ. Ureteroscopic results and complications: experience with 130 cases. F Urol 1988;139:710-3.

8. Seitz C, Liatsikos E, Porpiglia F, et al. Medical therapy to facilitate the passage of stones: what is the evidence? Eur Urol 2009;56:455-71, doi:10.1016/j.eururo.2009.06.012.

9. Hollingsworth JM, Rogers MA, Kaufman SR, et al. Medical therapy to facilitate urinary stone passage: a metaanalysis. Lancet 2006;368:1171-9, doi:10.1016/S0140-6736 (06)69474-9.

10. Singh A, Alter HJ, Littlepage A. A systematic review of medical therapy to facilitate passage of ureteral calculi. Ann Emerg Med 2007;50:552-63, doi:10.1016/j.annemergmed.2007.05.015.

11. Parsons JK, Hergun LA, Sukamoto K, et al. Efficacy of $\alpha$ blockers for the treatment of ureteral stones. 7 Urol 2007; 177:983-7, doi:10.1016/j.juro.2006.10.023.

12. Chan CW, Tekwani KL, Watts HF, et al. Emergency physicians report infrequent use of (alpha)-blockade for the treatment of ureteral stones. Am 7 Emerg Med 2009;27:7768, doi:10.1016/j.ajem.2008.06.007.

13. Stroup SP, Garvin AN, Irby J, et al. Practice patterns of primary care providers and urologists for use of medical expulsion therapy. Mil Med 2010;175:883-9.

14. Vincendeau S, Bellissant E, Houlgatte A, et al. Lack of efficacy of tamsulosin in the treatment of distal ureteral stones. Arch Intern Med 2010;170:2021-7, doi:10.1001/ archinternmed.2010.447.

15. Dellabella M, Milanese G, Muzzonigro G. Medical-expulsive therapy for distal ureterolithiasis: randomized prospective study on role of corticosteroids used in combination with tamsulosin - simplified treatment regimen and health-related quality of life. Urology 2005;66:712-5, doi:10.1016/j.urology. 2005.04.055.

16. Porpiglia F, Vaccino D, Billia M, et al. Corticosteroids and tamsulosin in the medical expulsive therapy for symptomatic 
distal ureter stones: single drug or association? Eur Urol 2006;50:339-44, doi:10.1016/j.eururo.2006.02.023.

17. Porpiglia F, Ghignone G, Fiori C, et al. Nifedipine versus tamsulosin for the management of lower ureteral stones. $7 \mathrm{Urol}$ 2004;172:568-71, doi:10.1097/01.ju.0000132390.61756.ff.

18. Dellabella M, Milanese G, Muzzonigro G. Efficacy of tamsulosin in the medical management of juxtavesical ureteral stones. 7 Urol 2003;170:2202-5, doi:10.1097/01.ju. 0000096050.22281.a7.

19. Dellabella M, Milanese G, Muzzonigro G. Randomized trial of the efficacy of tamsulosin, nifedipine and phloroglucinol in medical expulsive therapy for distal ureteral calculi. $7 \mathrm{Urol}$ 2005;174:167-72, doi:10.1097/01.ju.0000161600.54732.86.

20. Erkan E, Toktas GM, Kocaaslan R, et al. Efficacy of tamsulosin and deflazacort in management of distal ureteral stones: a prospective randomized trial. Eur Urol Suppl 2010; 9:581, doi:10.1016/S1569-9056(10)61380-2.

21. Higgins JPT, Green S, editors. Cochrane bandbook for systematic reviews of interventions. Version 5.1.0. 2011. Available at: www.cochrane-handbook.org (accessed June 30, 2013).

22. Porpiglia F, Fiori C, Ghignone G, et al. A second cycle of tamsulosin in patients with distal ureteric stones: a prospective randomized trial. BfU Int 2009;103:1700-3, doi:10.1111/j.1464-410X.2008.08295.x.

23. Ochoa-Gomez R, Prieto-Diaz-Chavez E, Trujillo-Hernandez B, et al. Tamsulosin does not have greater efficacy than conventional treatment for distal ureteral stone expulsion in Mexican patients. Urol Res 2011;39:491-5, doi:10.1007/ s00240-011-0380-z.

24. Abdel-Meguid TA, Tayib A, Al-Sayyad A. Tamsulosin to treat uncomplicated distal urerteral calculi: a double blind randomized placebo-controlled trial. Can $\mathcal{F}$ Urol 2010;17: 5178-83.

25. Ahmed AF, Al-Sayed AY. Tamsulosin versus alfuzosin in the treatment of patients with distal ureteral stones: prospective, randomized, comparative study. Korean 7 Urol 2010;51:1937, doi:10.4111/kju.2010.51.3.193.

26. Agrawal M, Gupta M, Gupta A, et al. Prospective randomized trial comparing efficacy of alfuzosin and tamsulosin in management of lower ureteral stones. Urology 2009;73:706-9, doi:10.1016/j.urology.2008.11.013.

27. Al-Ansari A, Al-Naimi A, Alobaidy A, et al. Efficacy of tamsulosin in the management of lower ureteral stones: a randomized double-blind placebo-controlled study of 100 patients. Urology 2010;75:4-7, doi:10.1016/j.urology.2009. 09.073 .

28. Aldemir M, Ucgul YE, Kayigil O. Evaluation of the efficiency of tamsulosin and Rowatinex in patients with distal ureteral stones: a prospective, randomized, controlled study. Int Urol Nephrol 2010;43:79-83.

29. Arrabal-Martin M, Valle-Diaz De La Guardia F, ArrabalPolo MA, et al. Treatment of ureteral lithiasis with tamsulosin: literature review and meta-analysis. Urol Int 2010;84:254-9, doi:10.1159/000288224.

30. Autorino R, De Sio M, Damiano R, et al. The use of tamsulosin in the medical treatment of ureteral calculi: where do we stand? Urol Res 2005;33:460-4, doi:10.1007/ s00240-005-0508-0.
31. Avdoshin VP, Andriukhin MI, Barabash MI, et al. Tamsulosin in the treatment of patients with ureteroliths of the lower third of the ureter clinical and pharmacoeconomic grounds. Urologiia 2005;(4):36-9.

32. Cervenakov I, Fillo J, Mardiak J, et al. Speedy elimination of ureterolithiasis in lower part of ureters with the alpha 1-blocker-tamsulosin. Int Urol Nephrol 2002;34:25-9, doi:10.1023/A:1021368325512.

33. De Sio M, Autorino R, Di Lorenzo G, et al. Medical expulsive treatment of distal-ureteral stones using tamsulosin: a single-center experience. $\mathcal{F}$ Endourol 2006;20:12, doi:10.1089/end.2006.20.12.

34. Erturhan S, Erbagci A, Yagci F, et al. Comparative evaluation of efficacy of use of tamsulosin and/or tolterodine for medical treatment of distal ureteral stones. Urology 2007; 69:633-6, doi:10.1016/j.urology.2007.01.009.

35. Ferre RM, Wasielewski JN, Strout TD, et al. Tamsulosin for ureteral stones in the emergency department: a randomized, controlled trial. Ann Emerg Med 2009;54:4329, doi:10.1016/j.annemergmed.2008.12.026.

36. Hermanns T, Sauermann P, Rufibach K, et al. Is there a role for tamsulosin in the treatment of distal ureteral stones of $7 \mathrm{~mm}$ or less? Results of a randomised, double-blind, placebo-controlled trial. Eur Urol 2009;56:407-12, doi:10.1016/j. eururo.2009.03.076.

37. Kupeli B, Irkilata L, Gurocak S, et al. Does tamsulosin enhance lower ureteral stone clearance with or without shock wave lithotripsy? Urology 2004;64:1111-5, doi:10. 1016/j.urology.2004.07.020.

38. Lojanapiwat B, Kochakarn W, Suparatchatpan N, et al. Effectiveness of low-dose and standard-dose tamsulosin in the treatment of distal ureteric stones: a randomized controlled study. 7 Int Med Res 2008;36:529-36, doi:10. 1177/147323000803600318.

39. Sayed MAB, Aboul-Yosr AM, Abdalla MA, et al. Efficacy of tamsulosin in medical expulsive therapy for distal ureteral calculi. Scand 7 Urol Nephrol 2008;42:59-62, doi:10.1080/ 00365590701571076.

40. Wang CJ, Huang SW, Chang CH. Efficacy of an alpha1 blocker in expulsive therapy of lower ureteral stones. 7 Endourol 2008;22:41-6, doi:10.1089/end.2007.0133.

41. Yilmaz E, Batislam E, Basar MM, et al. The comparison and efficacy of 3 different (alpha)1-adrenergic blockers for distal ureteral stones. 7 Urol 2005;173:2010-2, doi:10.1097/ 01.ju.0000158453.60029.0a.

42. Han MC, Park YY, Shim BS. Effect of tamsulosin on the expectant treatment of additive expulsion effect of lower ureteral stones. Korean 7 Urol 2006;47:708-11, doi:10.4111/ kju.2006.47.7.708.

43. Kim JW, Cho DY, Lee JG. Effect of tamsulosin on the expected treatment of upper and lower ureteral stones. Korean 7 Urol 2007;48:724-30, doi:10.4111/kju.2007.48. 7.724 .

44. European Association of Urology. Online guidelines. Available at: http://www.uroweb.org/guidelines/online-guidelines (accessed November 10, 2010).

45. Tintinalli JE, Kellen GD, Stapcsynski JS. Emergency medicine: a comprehensive guide. 6th ed. McGraw-Hill; 2004. 


\begin{tabular}{|c|c|c|}
\hline 2 & \#1 AND "tamsulosin"/exp & 214 \\
\hline 4 & $\begin{array}{l}\text { \#1 AND ("alpha adrenergic receptor blocking agent"'/exp OR "alpha antagonist" } \\
\text { OR "'alpha antagonists" OR "alpha-antagonist" OR "alpha-antagonists" OR } \\
\text { "alpha blocker"'lexp OR "alpha blockers" OR "alpha-blocker'"/exp OR "alpha- } \\
\text { blockers" OR "alpha receptor antagonist" OR "alpha receptor antagonists") }\end{array}$ & 490 \\
\hline 5 & \#1 AND alna & 1 \\
\hline 6 & $\# 1$ AND urolosin & 0 \\
\hline 11 & \#1 AND ('flomax"/exp OR flomax) & 0 \\
\hline 12 & \#2 OR \#3 OR \#4 OR \#5 OR \#6 OR \#7 OR \#8 OR \#9 OR \#10 OR \#11 & 491 \\
\hline
\end{tabular}

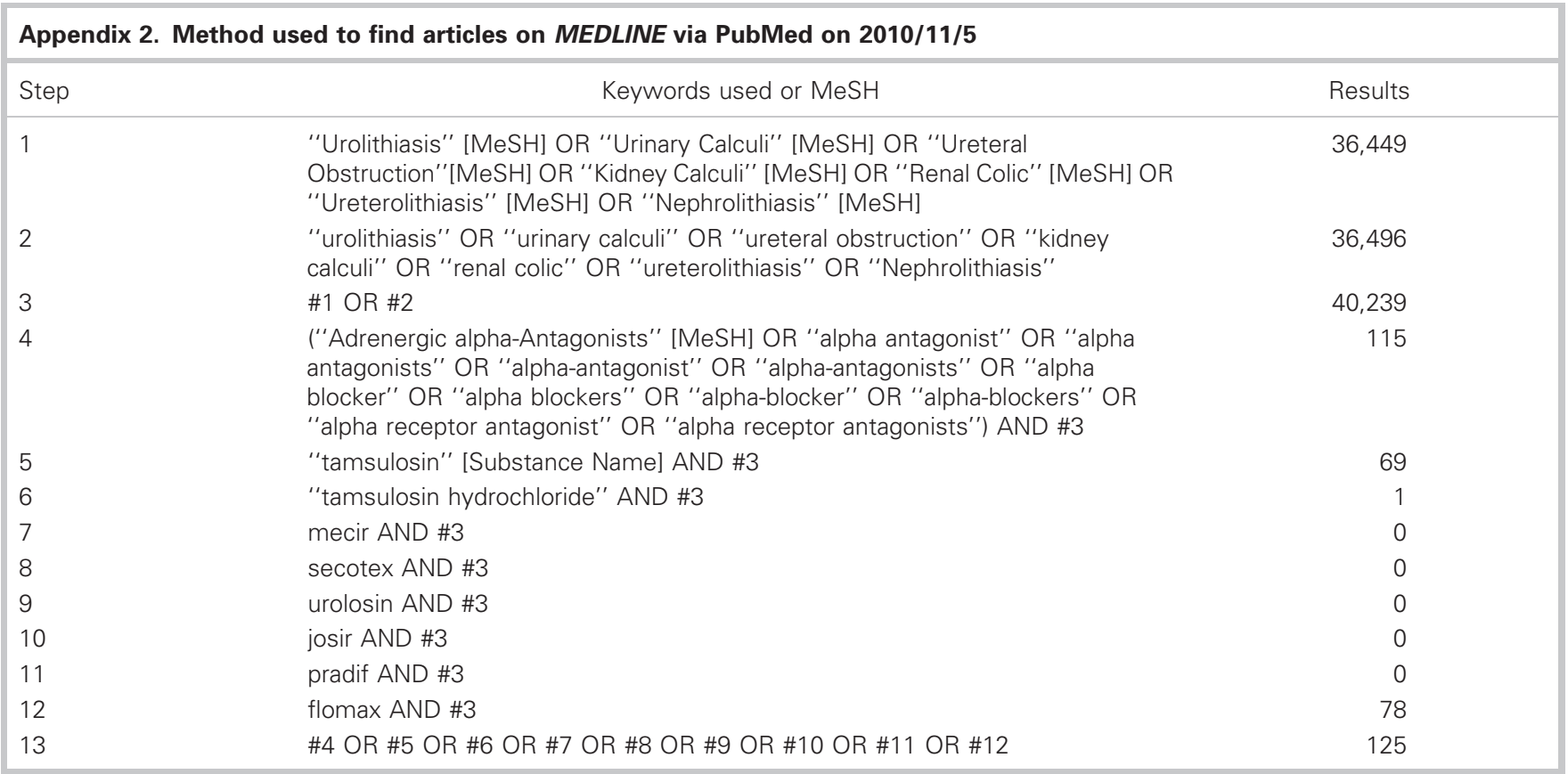




\begin{tabular}{|c|c|c|}
\hline 2 & MeSH descriptor Ureteral Calculi explode all trees & 233 \\
\hline 3 & MeSH descriptor Renal Colic explode all trees & 2 \\
\hline 4 & MeSH descriptor Ureterolithiasis explode all trees & 235 \\
\hline 7 & $\begin{array}{l}\text { "urolithiasis" OR "urinary calculi" OR "ureteral obstruction" OR "kidney } \\
\text { calculi" OR "renal colic" OR "ureterolithiasis" OR "Nephrolithiasis" }\end{array}$ & 855 \\
\hline 8 & \#1 OR \#2 OR \#3 OR \#4 OR \#5 OR \#6 OR \#7 & 1,008 \\
\hline 9 & (tamsulosin AND \#8) & 49 \\
\hline 10 & ("tamsulosin hydrochloride" AND \#8) & 1 \\
\hline 13 & $\begin{array}{l}\text { "flomax" OR "josir" OR "mecir" OR "urolosin" OR "secotex" OR "alna" OR } \\
\text { "pradif" AND \#8 }\end{array}$ & 0 \\
\hline 14 & \#9 OR \#10 OR \#11 OR \#12 OR \#13 & 58 \\
\hline 15 & \#12 AND restrict by product: Clinical Trials & 48 \\
\hline
\end{tabular}

\section{Appendix 4. Cochrane Collaboration's tool for assessing risk of bias}

Domain

Sequence generation

Allocation concealment

Blinding of participants, personnel, and outcome assessors (assessments should be made for each main outcome or class of outcomes)

Incomplete outcome data (assessments should be made for each main outcome or class of outcomes)

Selective outcome reporting

Other sources of bias
Description

Review authors' judgment

Was the allocation sequence adequately generated? Describe the method used to generate the allow an assessment of whether it should produce comparable groups.

Describe the method used to conceal the allocation sequence in sufficient detail to determine whether intervention allocations could have been foreseen in advance of, or during, enrolment.

Describe all measures used, if any, to blind study participants and personnel from knowledge of which intervention a participant received. Provide any information relating to whether the intended blinding was effective.

Describe the completeness of outcome data for each main outcome, including attrition and exclusions from the analysis. State whether attrition and exclusions were reported, the numbers in each intervention group (compared to total randomized participants), reasons for attrition/exclusions where reported, and any reinclusions in analyses performed by the review authors.

State how the possibility of selective outcome reporting was examined by the review authors and what was found.

State any important concerns about bias not addressed in the other domains in the tool.

If particular questions/entries were prespecified in the review's protocol, responses should be provided for each question/entry.
Was knowledge of the allocated intervention adequately prevented during the study?

Were incomplete outcome data adequately addressed?

Are reports of the study free of suggestion of selective outcome reporting?

Was the study apparently free of other problems that could put it at a high risk of bias? 